\title{
Hidrops vesicular en recién nacidos y lactantes
}

\author{
Dra. Karla Moüne B.; ${ }^{1}$ Dr. Germán Mühlhausen M.;2 Dr. Gustavo Aldunate N. 3
}

Hydrops of the gallbladder

Hydrops of the gallbladder was detected in three patients: the first one was a 4 day old preterm newborn witl clinical evidence of haline membrane disease, the other two cases occurted in infants aged 1 and 2.5 months and were pricoded by acute diarrhea. The first evidence of gallbladder's hydrops was a palpable mass at the riglit upper abdaminal quadrant in all cases. Diagnoses were confinned by abdominal ultasonography; spontancous iemission oceured when the initial discase becane under tontrol.

(Key words: hydrops, gallbladder, hyaline mombrane disease, acute diarrhea, abdominal mass, ultrasonography).

Al enfrentarse a un niño con masa abdominal palpable, el médico clínico puede orientar su diagnóstico sobre la base de caracteristicas del examen físico, síntomas asociados y exámenes de laboratorio en los cuales juega un rol fundamen. tal el diagnóstico por imágenes. Un diagnóstico adecuado es básico para un tratamiento, dado que no siempre la presencia de una masa abdominal en cste grupo etario, es indicación de tratamiento quirúrgico.

Las causas de masa abdominal palpable del cuadrante superior derecho del abdomen en recién nacidos y lactantes son variadas: hidrone-

1. Unidad de Radiología e Jnagenes, Hospital Félix Bujnes Cerda.

2. Unidad de Neonatología, Hospital Félik Bulnes Cerda.

3. Servicio de Cirugia Infantil y Ortopedia, Hospital Roherto del Río. frosis, rin̄ón multiquístico, tumor renal, rînones poliquísticos, hemorragia suprarrenal, quiste del colédoco, tumores hepáticos, neuroblastoma, etc.

El hidrops vesicular (HV) constituye una causa no habitual de masa abdominal palpable en recién nacidos y lactantes menores, razón por la cual no se plantea habitualmente como posibili\{ad diagnóstica inicial. ${ }^{1.14}$

EI HV es una distensión alitiásica no inflamatoria de la vesicula biliar cuyo diagnóstico de certezá se efectúa con ultrasonografía (US), ${ }^{1,}, 6$, 7 resultando en ocasiones de alguna utilidad el estudio con radiografia de abdomen simple.6,8 La colecistografía y estudio cintigráfico de la vía biliar, han resultado de rendimiento limitado en esta afección. ${ }^{6,9}$

\section{Casos clínicos}

1. Recién nacido de sexo masculino, producto de una cesárea por hipertensión arterial severa. Al 
nacer pesó $2.270 \mathrm{~g}$ y obtuvo un puntaje de Apgar de 2(1), 5(3) y 8(10). Fue hospitalizado en la unidad de recién nacidos con los diagnósticos de: recién nacido pre-término (RNPT) adecuado a su edad gestacional (AEG), 36 semanas de gestación; asfixia perinatal grave recuperada; sindrome de dificultad respiratoria; hijo de madre diabética. Ingresó con quejido, polipnea, cianosis y retracción subcostal e intercostal. evidencia clínica y radiológica de enfermedad de membrana hialina que requirió ventilación asistida durante 30 horas y luego presión continua en la vía aérea (CPAP) por 36 horas adicionales. El hemograma al $2^{\circ}$ día de vida resultó normal Al $4^{\circ}$ día de edad se palpó una masa en el hipocondrio derecho la que se interpretó como descenso hepático. Concomitantemente a este hallazgo se observó hiperbilirrubinemia de predominio indirecto (10 $\mathrm{mg} / \mathrm{dL}$ ), sin incompatibilidad de grupo sanguíneo, que requirió tratamiento con Juminoterapia durante 4 días. Al $6^{\circ}$ día de vida persistía la masa palpable antes descrita. La ultrasonogra. fía abdominal demost ró que correspond ía a vesicula distendida de $38 \mathrm{~mm}$ de longuitud. Al día siguiente un nuevo control ultrasonográfico no demostró variaciones. Al $10^{\circ}$ día de edad clínicamente no pudo palparse esta masa $y$ ultrasonográficamente la vesícula biliar fue de características normales. Se dió de alta al $11^{\circ}$ día on buenas condiciones.

2. Paciente de sexo masculino, producto de parto gemelas en podálica. Al naces pesó $2.050 \mathrm{~g}$ y ubtuvo calificación de Apgar de 7(1 ) y 9(5). Fue hospitalizado en la unidad de neonatología con los diagnósticos de: RNPT AEG 33 a 34 semanas de gestación, y segundo gemelar. Durante su estadia tuvo hiperbiimubinemia de predominio indirecto que requirió luminoterapja durante ei $4^{\circ}$ y el $5^{\circ}$ dia de vidia. Fue dado de alta a la edad de 7 dias en buenas condiciones. Reingre. só a la edad de un mes a la unidad de lactantes a causa de diarre aguda, deshidratación $y$ acidosis. En los exámenes de ingreso se detectaron leuco. citos fecales $+++y$ en el hemograma habian 6000 leucocitos $\mathrm{x} \mathrm{mm^{3 }}, 22 \%$ baciliformes y $8 \%$ segmentados.

Se suministró furazolidona oral y líquidos, incluyendo bicarbonato de sodio por vía endovenosa para cubrir requerimientos y reparar sus alteraciones hidroelectroliticas y acidobásicas.

Al tercer día de hospitalización se palpó una masa en el hiponcondrio de recho de $2 \times 2 \mathrm{~cm}$, de consistencia finme y superficic lisa. El hemo. grama de ese dia mostró un recuento de leucocj. tos de $8900 \times \mathrm{mm}^{3}$ con $13 \%$ de baciliformes. La bilirrubinemia fue de $6,5 \mathrm{mg} / \mathrm{dL}$ con $2,1 \mathrm{mg} / \mathrm{dL}$ de bilirrubinemia directa ( $32 \%)$, transaminasas pirúvicas de 35 U/L. En la radiografía de abdomen simple se veia una imagen sugerente de masa en el hipocondrio derecho (figura 1). Con estos antecedentes se inicio tratamiento endovenoso con ampicilina y gentamicina en base a los diagnósticos de síndrome diarreico agudo con deshidratación y hepatitis séptica.

La ultrasonografía abdominal del día siguiente demostró correspondencia de la masa palpable con una vesicula biliar muy distendida y acodada, cuya longitud fue de aproximadamente 56 mm.

En el $6^{\circ}$ dia de hospitalización la bilirrubinemia era $3,5 \mathrm{mg} / \mathrm{dL}$ con $2,7 \mathrm{mg} / \mathrm{dL}$ de indirecta, las transaminasas pirúvicas $36 \mathrm{U} / \mathrm{L}$ y las fosfatasas a lcalinas 15,8 UB. En la ultrasonografía abdominal realizada 10 dias después de la anterior, la vesícula biliar parecía normal y se observaba una

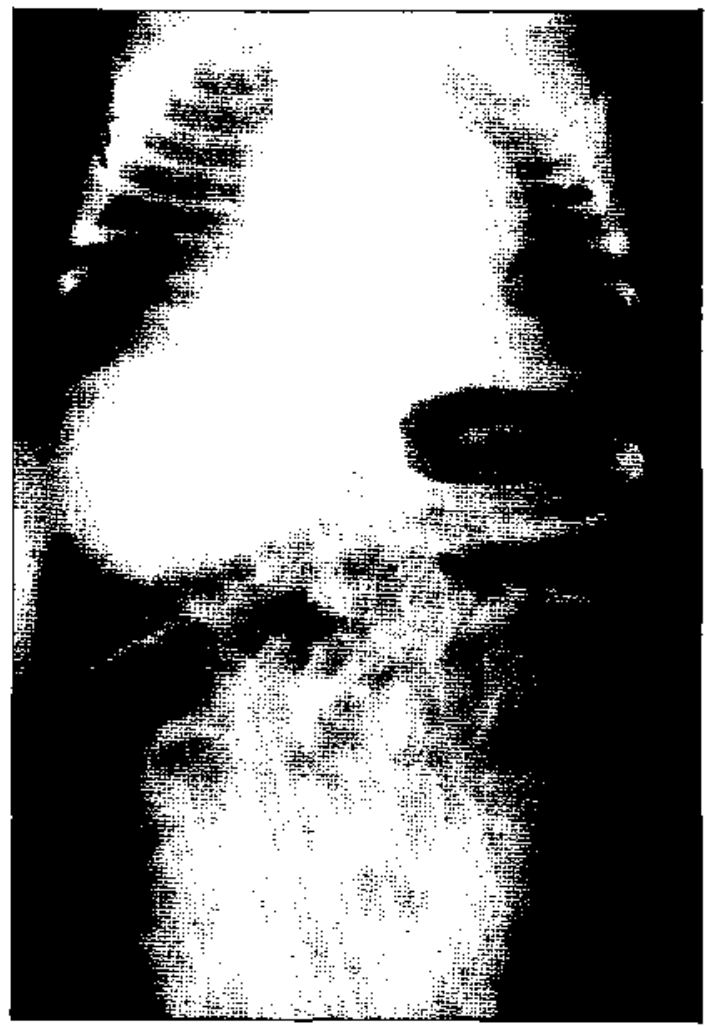

Figura 1: Radioyafía toracoubdominal simple en el waso 2 mostrando una masa en el hipocondrio derecho. 
leve hidronefrosis derecha. $\mathrm{Al}$ cabo de $\mathrm{C} 0$ días $\mathrm{dc}$ tratamiento $y$ con hemocultivos negativos. se suspendieron los antibióticos. Exámenes posteriores demostraron que este paciente tenía estenosis pieloureteral derecha leve o infección urinaria, con urocultivo de Klebsiella sp. que se trató con amikacina con buenos resultados. Aunque antes del alta sufrió diarrea causada por Shigella flexneri y bronconcumonia, se recuperó y fue dado de alta a la edad de 4 meses y 16 dias en buenas condiciones.

3. Paciente de sexo masculino nacido de parto eutócico. Al nucer pesó $1.700 \mathrm{~g}$ y fue hospitali. zato en Ja unidad de neonatologja durante una scmana en incubadora con luminoterapia por hiperbilirrubinemia $\sin$ incompatibilidad de grupo sanguínco. Reingresó a la unidad de lactantes a la calad de 2 meses y 23 días por síndrome diarreico agudo con deshidrataciôn y síndrome bronquial obstructivo.

Se trató con réginen de ayuno y solución glucosulina endovenosa. Desde el segundo día la solución hidroclectrolítica se administró mediante gastroclisis. El mismo día aparccieron decajmiento, palidez terrosa y hepatoesplenomegalia, efectuándose punción lumbar, sedimento le orjna, urocultivo, y una radiografia de tórax que mostraba signos de bronconeumonia derecha por lo que se trató 7 dias con penicilina sódica.

Al tercer día por intolerancia a la lactosa fue tratada con la dieta apropiada con lo que se normalizaron rajpidamente las deposiciones. $\Lambda \mathrm{i}$ cuar to día de hospitalización apareció una masa palpable en el hipocondrio derecho de $2 \times 2 \mathrm{~cm}$ que en ultrasonografia abdominal correspondia a hidrops vesicular (vesicula $=65 \mathrm{~mm})($ figura $2 a)$. Las mediciones de bilirrubinemia, protrombinemia y transaminasa piruvica resultaron normales. En el hemograma había leucocitos con predominio de linfocitos.

La ultrasonografía abdominal de control, 4 dáas después de la anterior, demostró regresión del tamaño vesicular (1igura 2b) y normalización cn e] examen ultrasonográfico practicado a los 10 dias (figura 2c).

\section{DISCUSION}

La etiologia del HV en el recién nacido y en el lactante menor no ha sido totalmente aclarada, planeándose como causas probables el espesa-
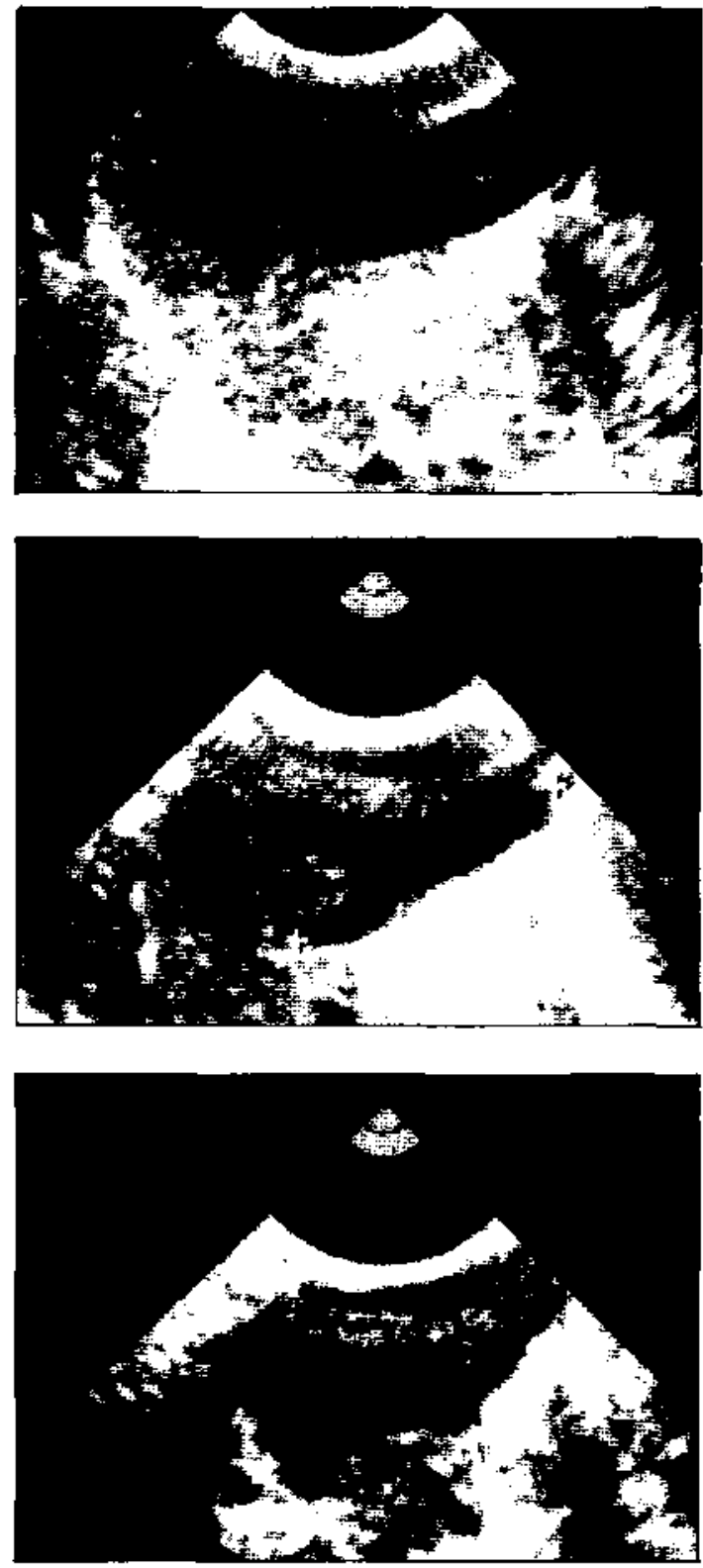

Figura 2: Ultrasonografía del caso 3: a) en el cuadrante superior derecho de la fotografia se ubserva imagen sonolúcida que cor responde a hidrops vesicular. b) y c) reducción y deraparición de la imagen descrita.

miento transitorio de la bilis en el interior de la vía biliar, acodaduras del conducto cístico, comprensión del conducto cístico por ganglios linfáticos aumentados de tamaño y reacción inflamatoria del conducto cístico a partir de 
adenitis mesenterica. ${ }^{b}+10$ Se ha comunicado además hidrops posterior a infecciones del tracto respiratorio superior, gastroenteritis, hepatitis infecciosa, escarlatina, leptosporosis, diarrea y linfadenopatía cervica]. $6,8,11,12$

De nuestros tres casos, dos (casos 2 y 3) fueron precedjdos por un episodio de diarrea aguda con deshidratación. Se ha involucrado en la patogenia del $\mathrm{HV}$ en estos casos el espesamiento transitorio de bilis secundario a la deshidratación 0 at régimen de ayuno con que se tratarl estos pacientes, que permitiría acumula. ción de bilis en la vesicula biliar ante un esfinter de Oddi cerrado. ${ }^{12}$ No bubo antecedentes de infección precedente $o$ desencadenante en el reciên nacido de esta serie.

$\mathrm{E} 1 \mathrm{HV}$ se describe en ambos sexos, pero más a menudo en varones como sucedió en estos tres pacientes y en el recién nacido.

Ocasionalmente puede haber fiebre, vómitos y deshidratación. Un signo importante to cons. tituyc la aparición de masa palpalble en hipocondrio derecho, $6,8,11,13$ observada en todos nuest ros pacientes.

Los síntomas y signos pueden simular apendicitis aguda, invaginación o peritonitis. ${ }^{6,8}$

La radiografía de abdomen simple puede sugerir masa en hemiabdomen derecho o no mostrar hallazgos de importancia. ${ }^{6,8}$ Mediante ultrasonografía puede observarse una masa piriforme en el cuadrante superior derecho, de contenido sonolúcido, que se separa del hígado y del riñón derecho y corresponde a la vesícula biliar de tamaño aumentado. Este es normalmente en el recièn nacido de 5 a $30 \mathrm{~mm}$ (promedio $15,2 \pm$ $7,4 \mathrm{~mm}$ DE). ${ }^{9,14}$ La ausencia de cálculos y la presencia de conductos biliares normales indica que esta masa corresponde a una distensión alitiásica de la vesícula biliar. El diagnóstico diferencial ultrasonográfico incluye quistes de colédoco (tipo III) y mesentéricos. ${ }^{14}$

El tratamiento de esta afección es médico, orientado a la enfermedad de base y a la infección cuando existen. La exploración quirúrgica en el HV debería estar reservada sólo para casos con evidencias de empiema vesicular (fiebre, aumento de la sensibilidad abdominal, leucocitosis, desviación a la izquierda y modifjcación del aspecto del contenido líquido vesicular en la sonografía), debiendo el cirujano, en la operación, considerar la necesidad de extirpar la vesi. cula o efectuar colesistostomía.

En la evolución de nuestros pacientes como los descritos en la literatura, la vesícula biliar volvió a su tamaño normal dentro de los 15 días siguientes al diagnóstico. ${ }^{3}$

\section{RESUMEN}

Se presentan tres casos de hidrops vesicular, un recién nacido y dos lactantes menores, en to que la uitrasonografía fue fundamental en el diagnóstico y seguimiento. Se analiza el cuadro clínico de esta patologia, que consiste en irritabjlidad, vómitos, deshidratación, ocasionalmente fiebre $y$ aparición de masa palpable abdominal, siendo este último hallazgo el más constante en estos pacientes. Se preconiza el tratamiento médico del hidrops vesicular, incluyendo el tratamiento de la infección concomitante cuando ésta exista.

\section{REFERENCIAS}

1. Gosik B., Leopold G.: UItrasound and the Gallblddder. Semin Roctgenol 1976; 11: 185-189.

2. Robinson A., Enwin I., Wiseman H., Kodroff M.: Cholecystitis and Hydrops of the Gallbladder in the newborn. Radiology 1977; 122: 749-751.

3. Rumley T.O., Rodgers B,M.: Hydrops of the Gallbledder in children. Pediatr Surg 1983; 18: 138.140 .

4. Neu J., Arvin A., Ariggno R: Hydrops of the Gallbladder. Am J Dis Child 1980; 134 : 891-893.

5. Jaden $M$., Alford B., Young L.: Radiclouical case of the month. Am J Dis Child 1984; $138: 985-986$.

6. Kumari S., Lee $W$., Baron M.: Hydtops of the Gallbladder in a Child Diagnosis by Uttrasonography. Pediatrics 1979; 63: 295-297

7. Appteby G., Forestier E., Stark C.: Hydrops of the Gallbladder in the neonatal period, Acta Paediat Scan 1981; 70: 117.120.

8. Scobie $\boldsymbol{W}$., Bentley J.: Hydrops of the Gallbladder in a newborn infant. J Pediatt Sury 1969; 4: 457-459.

9. Amodio I, Fontanetta E., Cohen M., Rudausky A. Rosenzweig M.: Neonatai hydrops of the Gallbladuer. N.Y. State J Med 1985; 85:565-566.

10. Hoyden C., Swischtick L.E.: Pediatric Ultrasonography. Baltimore Willians and Wilkins, 1987; 187-193.

11. Goldthorn J.F., Thomas D.W., Ramos A.D.: Hydrops of the Gallbladder in stressed prenature jufants. Clin Res 1980;28:122 A

12. Barth R.A., Erach R.C., Filfy R.A.- Abdominal pseudo-tumor in childbood: distended galloladder with parenteral hyperalimentation. AJR 1981; 136: 341-343.

13. Schrumpf J., Handmaker $H$.: Hydrops of Gallbladder in a Premature Neonate. Am J Dis Chil 1982 136: 172-173.

14. Bowen $A$.: Hydrops of the Gallbladder and choielithissis in neonates. Acta Paediatr Scand 1982; 71 : $839-841$. 